\title{
Abundancia y riqueza de hongos micorrizógenos arbusculares en cafetales de Soconusco, Chiapas, México
}

\author{
Vincenzo Bertolini ${ }^{1 *}$, Noé Manuel Montaño*2, Eduardo Chimal Sánchez ${ }^{2,4}$, \\ Lucía Varela Fregoso ${ }^{2}$, Jaime Gómez Ruiz ${ }^{1}$ \& José Martín Martínez Vázquez ${ }^{3}$ \\ 1. El Colegio de la Frontera Sur (ECOSUR), C.P. 30700, Tapachula, Chiapas, México; vin.bertolini@gmail.com, \\ jgomez@ecosur.mx \\ 2. Departamento de Biología, División de Ciencias Biológicas y de la Salud, Universidad Autónoma Metropolitana- \\ Iztapalapa, C.P. 09340, Ciudad de México, México; nmma@xanum.uam.mx, chimalse31@gmail.com, \\ lvarela@ipn.mx \\ 3. Universidad Politécnica de Tapachula (UPT), C.P. 30700, Tapachula, Chiapas, México; \\ martin.martinez.up@gmail.com \\ 4. Carrera de Biología, Facultad de Estudios Superiores Zaragoza, Universidad Nacional Autónoma de México, 09230, \\ Ciudad de México, México. \\ * Correspondencia
}

Recibido 29-V-2017. Corregido 19-X-2017. Aceptado 13-XI-2017.

\begin{abstract}
Abundance and richness of arbuscular mycorrhizal fungi in coffee plantations from Soconusco, Chiapas, Mexico. In the Mexican economy, and especially in the Soconusco region of Chiapas, coffee is economically important, and sustainable management most cover pests and diseases. In this study, we searched for native arbuscular mycorrhizal fungi (AMF) that could be used as biofertilizers in coffee cultivation. We collected 21 soil samples from seven coffee plantations (Coffea canephora) in November 2015. We used wet sieving and decanting to quantify abundance, richness and composition of morphospecies, as well as their relationships with soil properties. A total of 20 morphospecies and five new records of AMF were obtained, and the most frequent genera were Acaulospora and Glomus. The Toluca and Victoria sites had more morphospecies richness (17 spp. each) than San Agustín, November 20 and San Luis Nexapa (4-7 spp. each). Providencia and Platanar were intermediate but had the highest spore abundance. The dissimilarity of Victoria and Toluca may reflect a low concentration of $\mathrm{PO}_{4}^{-3}$ in the soil. $\mathrm{P}$ availability, linked to soil acidity, sems to be regulating these mycorrhizae communities at Soconusco. AMF consortia specific for soil P-levels and acidity, i.e. Acaulospora and Glomus consortia, may occur and be common in the environmental conditions of Mexican coffee plantations, Strains should be tested for compatibility and functionality before using them as native biofertilizers. Rev. Biol. Trop. 66(1): 91-105. Epub 2018 March 01.
\end{abstract}

Key words: arbuscular mycorrhizae, coffee plantations, fungal spores, soil phosphorous.

México exporta el $5 \%$ del volumen mundial de granos de café y está entre los ocho países latinoamericanos que concentran el $86 \%$ de la producción de café junto con Colombia, Guatemala, Costa Rica, Honduras, El Salvador, Nicaragua y Perú (ICO, 2016a-b). En la década de los 90, México ocupaba el tercer lugar como productor de café a nivel mundial, y a la fecha está en el décimo puesto (CEDRSSA, 2014; ICO, 2016a-b). Actualmente, este cultivo es el séptimo con mayor superficie cultivada en México y el doceavo como generador de divisas (SHCP, 2014). Los estados de Chiapas, Veracruz y Puebla contribuyeron con el $88 \%$ de la producción del café durante 2012 (Flores, 2015). Chiapas ha sido, en los últimos años, el principal productor mundial de café orgánico de altura (Barrera \& Parra, 2000). Además, la cafeticultura es una actividad con relevancia económica para la población indígena 
y campesina del centro y sureste de México (Fonseca, 2006). Por ejemplo, Mariscal (2011) reportó una producción de 18 millones de toneladas anuales, por más de 60000 productores, de los cuales una tercera parte eran mujeres indígenas y campesinas que orgánicamente cultivan los cafetos bajo sombra de árboles nativos.

En los últimos años este cultivo ha sido dañado por infestación de roya, enfermedad provocada por el hongo Hemileia vastatrix Berk. y Broome (FAO, 2015). Con porcentajes variables, casi toda la región del Soconusco en Chiapas es susceptible al ataque de este patógeno (DGSV-CNRF, 2015). Esta situación ha provocado la conversión de cafetales de la especie arábica (Coffea arabica L.) a cafetales de robusta (Coffea canephora L.), donde las condiciones climáticas y la altitud lo permiten, debido a que teóricamente esta variedad es menos susceptible $y$, en consecuencia, permite asegurar la producción y ganancia. Una alternativa a la conversión de cafetales y a la infestación por roya es el empleo de biofertilizantes que contengan microrganismos nativos que mejoren el estatus nutricional y de defensa de las plantas de este cultivo, tal es el caso de los hongos micorrizógenos arbusculares (HMA, Glomeromycota), los cuales mejoran el desarrollo de las plantas de café (Vaast, Zasoski, \& Bledsoe, 1997; de Almeida, Nogueira, Guimaraes \& Mourao, 2003; Andrade, Mazzafera, Schiavinato, \& Silveira, 2009; Trejo, Ferrera-Cerrato, García, Varela, Lara, \& Alarcón, 2011; Adriano, Jarquín, Hernández, Figueroa, \& Monreal, 2011). Los HMA se asocian con más del $80 \%$ de las plantas y forman en las raíces la micorriza arbuscular (Smith \& Read, 2008), la cual mejora la nutrición mineral y absorción de agua de la planta hospedera, especialmente cuando estos recursos son escasos en el suelo, además de protegerla del ataque de fitopatógenos de las raíces (Smith \& Read, 2008; Cuenca, 2015).

Los cafetos de sombra, por su comprobado bajo impacto sobre la biodiversidad, albergan en su rizosfera casi la totalidad de las especies nativas de los bosques sin perturbar (Arias,
Heredia, Sosa, \& Fuentes-Ramírez, 2012). Heredia y Arias (2008) indican que los cafetales de altura, con policultivo o árboles de sombra, albergan más diversidad de HMA que los bosques nativos, reportando hasta 79 especies de HMA en plantaciones de Coffea arabica en Veracruz, México; en donde el tipo de manejo afecta fuertemente a las comunidades de HMA (Posada, Sánchez de Prager, Heredi, \& Sieverding, 2016). No obstante, a pesar de que se han examinado los HMA en algunas plantaciones de café, su riqueza y composición podría diferir de otras plantaciones, ya que las condiciones edáficas modifican las comunidades de HMA (de Beenhouwer, Van Geel, \& Ceulemans, 2015; Posada et al., 2016). Así, detectar estos factores puede ser crítico en la selección de consorcios de HMA efectivos en las plantaciones de café en México (Trejo et al., 2011).

En México, las aplicaciones de HMA a varios tipos de cultivo, entre ellos el del café, han favorecido su desarrollo y rendimiento (Aguirre-Medina, 2006; Trejo et al., 2011; Adriano et al., 2011). El efecto benéfico de los HMA se define en función de su efectividad en el crecimiento y/o nutrición de la planta (Trejo et al., 2011); sin embargo, esto depende de la compatibilidad entre el hongo y la planta (Van der Heijden \& Sanders, 2002). En consecuencia, los inóculos serán más efectivos cuanto más compatibles sean con la planta hospedera. En el estado de Chiapas poco se ha investigado al respecto $\mathrm{y}$, en particular en la región del Soconusco, se carece de estudios específicos acerca de la abundancia, riqueza y composición de los HMA, información que es crucial para el desarrollo de inoculantes micorrízicos compatibles con el cultivo del café. Con el fin de promover la producción sustentable del café a través de alternativas a la fertilización convencional, se está llevando a cabo una investigación básica para buscar los HMA nativos asociados a los cafetales, que pudieran emplearse, en un futuro, como cepas micorrízicas base para preparar biofertilizantes que, eventualmente, se apliquen a los cultivos de café, con la finalidad de impactar positivamente su producción (Adriano et al., 2011). 
Esto debido a que se ha reportado que la fertilización química reduce la riqueza y abundancia de HMA en los sistemas cafetaleros (Arias et al., 2012, de Beenhouwer et al., 2015). Por lo tanto, el objetivo de este trabajo fue determinar la abundancia de esporas y la riqueza y composición de morfoespecies de HMA en suelo rizosférico de plantas de cafetos y asociarlos a las características edáficas en la región del Soconusco, Chiapas, México.

\section{MATERIALES Y MÉTODOS}

Área de estudio: El área en donde se localizan los sitios cafetaleros elegidos para este estudio está en la región del Soconusco al sur del estado de Chiapas, México, que se ubica entre los (15¹9'00” N \& 92 $44^{\prime} 12^{\prime \prime}$ W), cubriendo $5475 \mathrm{~km}^{2}$ del territorio de Chiapas. El tipo de vegetación predominante hoy en día son los fragmentos de la selva perennifolia y del bosque mesófilo de montaña, rodeados por zonas agrícolas, cultivos de café y bosque secundario. Los principales tipos de suelo son Nitosoles y Cambisoles (INIFAP, 2010). El clima es cálido húmedo y/o subhúmedo con abundantes lluvias en verano. La temperatura media anual varía de 21 a $26{ }^{\circ} \mathrm{C}$ y la precipitación total anual está en el intervalo de los 2290 a $4614 \mathrm{~mm}$ (CONANP, 2011).

Sitios de recolecta: En noviembre del 2015, se recolectaron muestras de suelo rizosférico asociado a plantas de cafeto (Coffea canephora), en siete diferentes sitios de zonas cafetaleras localizadas en la región del Soconusco (Cuadro 1, Fig. 1) y que abarcaron altitudes entre 400 y $1300 \mathrm{~m}$. En cada sitio se recolectaron tres muestras simples de suelo de aproximadamente $300 \mathrm{~g}$ cada una, obtenidas al pie de tres diferentes cafetos a una profundidad de $20 \mathrm{~cm}$ y a una distancia aproximada de 100

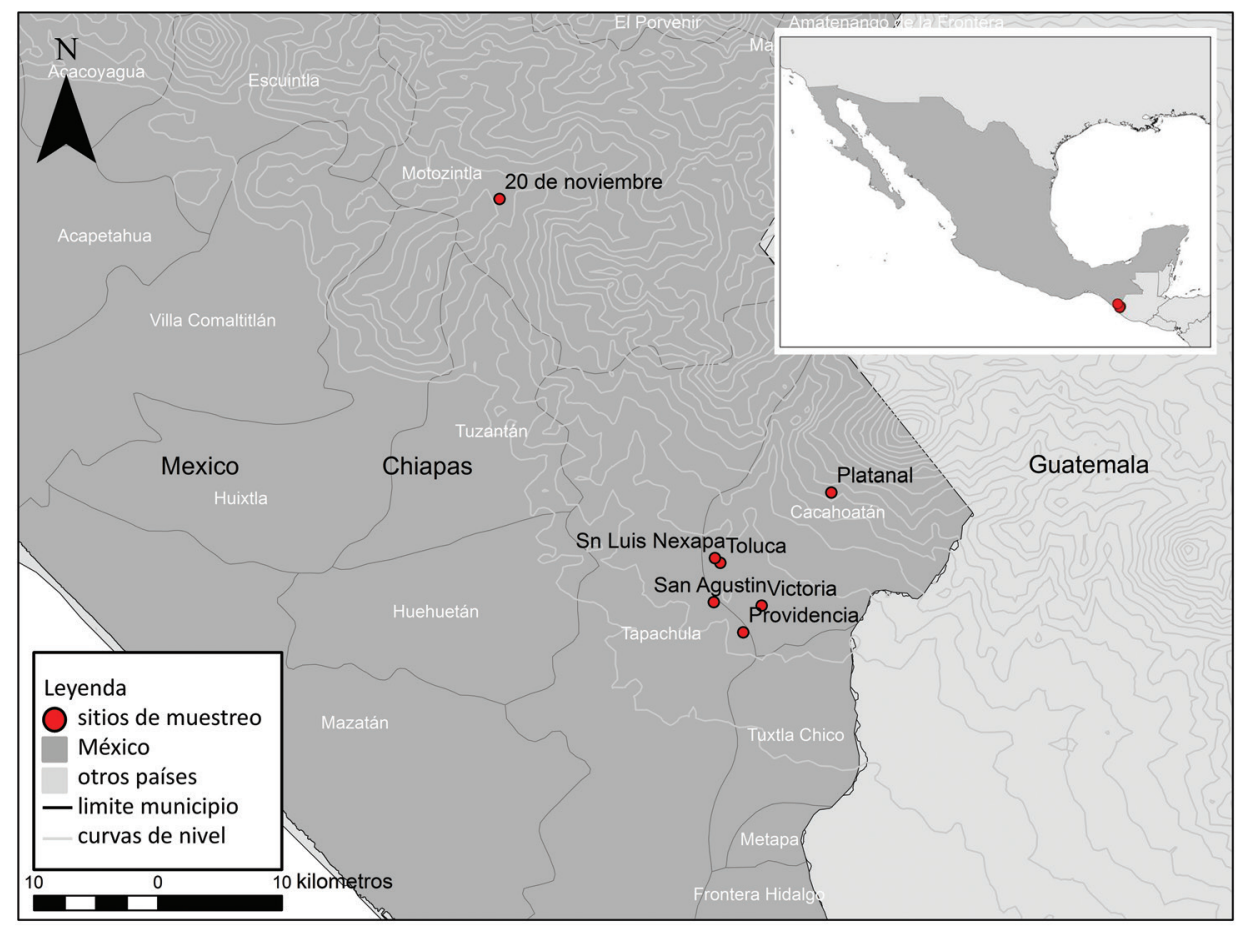

Fig. 1. Fincas cafetaleras en la región del Soconusco, Chiapas, México, en donde fue recolectado suelo rizosférico de Coffea canephora para la obtención de esporas de hongos micorrizógenos arbusculares para este estudio.

Fig. 1. The sites in coffee farms at the Soconusco region, Chiapas, Mexico, where rhizospheric soil of Coffea canephora was collected to obtain spores of arbuscular mycorrhizal fungi for this study. 
CUADRO 1

Localización y parámetros climáticos de los sitios cafetaleros en la región del Soconusco, Chiapas, México, en donde fue recolectado suelo rizosférico de Coffea canephora para la obtención de esporas de hongos micorrizógenos arbusculares

TABLE 1

Location and climatic parameters of sites with coffee plants at the Soconusco region, Chiapas, Mexico, where rhizospheric soil associated to Coffea canephora was collected to obtain the spores of arbuscular mycorrhizal fungi

\begin{tabular}{lccccc}
\multicolumn{1}{c}{ Sitio/finca } & Latitud* & Longitud* & $\begin{array}{c}\text { Altitud } \\
(\mathrm{m} \mathrm{snm})\end{array}$ & $\begin{array}{c}\text { Temperatura } \\
\text { media anual }\left({ }^{\circ} \mathrm{C}\right) * *\end{array}$ & $\begin{array}{c}\text { Precipitación anual } \\
(\mathrm{mm}) * *\end{array}$ \\
Platanar & $15^{\circ} 5^{\prime} 10.31^{* \prime} \mathrm{N}$ & $92^{\circ} 9^{\prime} 44.28^{\prime \prime} \mathrm{W}$ & 1320 & 21.4 & 3971 \\
Victoria & $15^{\circ} 0^{\prime} 15.36^{\prime \prime} \mathrm{N}$ & $92^{\circ} 12^{\prime} 46.17^{\prime \prime} \mathrm{W}$ & 463 & 26.1 & 4491 \\
Providencia & $14^{\circ} 59^{\prime} 5.81^{\prime \prime} \mathrm{N}$ & $92^{\circ} 13^{\prime} 34.16^{\prime \prime} \mathrm{W}$ & 462 & 26.1 & 4451 \\
San Agustín & $15^{\circ} 0^{\prime} 24.46^{\prime \prime} \mathrm{N}$ & $92^{\circ} 14^{\prime} 51.00^{\prime \prime} \mathrm{W}$ & 446 & 26.2 & 4379 \\
Toluca & $15^{\circ} 2^{\prime} 7.04^{\prime \prime} \mathrm{N}$ & $92^{\circ} 14^{\prime} 34.11^{\prime \prime} \mathrm{W}$ & 540 & 25.8 & 4614 \\
San Luis Nexapa & $15^{\circ} 2^{\prime} 19.68^{\prime \prime} \mathrm{N}$ & $92^{\circ} 14^{\prime} 48.02^{\prime \prime} \mathrm{W}$ & 617 & 25.8 & 4614 \\
20 de noviembre & $15^{\circ} 17^{\prime} 55.42^{\prime \prime} \mathrm{N}$ & $92^{\circ} 24^{\prime} 9.95^{\prime \prime} \mathrm{W}$ & 1069 & 23.5 & 2292 \\
\hline
\end{tabular}

Nota: *Unidades sexagesimales; m snm= metros sobre el nivel del mar; ** Hijmans et al. (2005), http://www.worldclim.org/

$\mathrm{m}$ entre sí. Con el objetivo de encontrar abundante esporulación, se llevó a cabo el muestreo durante la época de más escasa precipitación, que corresponde a los meses de octubrediciembre (Cuenca \& Lovera, 2010). El manejo de los cafetales de donde se recolectaron las muestras es de bajo recurso tecnológico, con régimen de sombra parcial [realizada a través de podas a los árboles de sombra empleados (Inga spp.)], sin plan ni sistema de fertilización, sin deshierbes manuales, sin aplicaciones de agroquímicos, y en donde se obtiene un promedio de producción de grano por hectárea de una tonelada por año (Moguel \& Toledo, 1999). Las plantaciones se seleccionaron en tales condiciones con la idea que el suelo fuese lo más conservado posible y así garantizar la presencia en condiciones de campo de HMA nativos.

Extracción de las esporas de HMA: Las esporas de HMA se extrajeron con la técnica de tamizado húmedo y decantación (Gerdemann \& Nicolson, 1963) modificada por Brundrett, Bougher, Dell, Grove y Malajczuk (1996). Así, para cada una de las muestras de suelo recolectadas se pesaron $100 \mathrm{~g}$ de suelo seco, se adicionaron $400 \mathrm{~mL}$ de agua y se realizó una suspensión mediante agitación mecánica para romper los agregados del suelo; posteriormente la suspensión se pasó en tamices con malla de 1000 y 44 micras a un mismo tiempo. La muestra de suelo retenida en los tamices se colocó en dos tubos $(50 \mathrm{~mL})$, los cuales fueron centrifugados a $2500 \mathrm{rpm}$ por $5 \mathrm{~min}$. El sobrenadante fue eliminado y la muestra de suelo retenida se re-suspendió en otros dos tubos que contenían una solución de sacarosa al $60 \%$ y se centrifugaron nuevamente a 1 $200 \mathrm{rpm}$ por $5 \mathrm{~min}$ recuperando el sobrenadante en el tamiz de $44 \mu \mathrm{m}$. Se usó una caja de Petri dividida en cuadrantes $(0.5 \times 0.5 \mathrm{~cm})$ para extraer las esporas de HMA, considerando solo aquellas que presentaron contenido y coloración homogénea. Las esporas se colocaron en preparaciones permanentes, usando alcohol polivinílico en lactoglicerol (PVLG) y PVLG + reactivo de Melzer (1:1), según lo recomendado por la International Culture Collection of (Vesicular) Arbuscular Mycorrhizal Fungi (INVAM, 2016). Las esporas se observaron en un microscopio óptico (Iroscope mod. ES-24®) para determinar su abundancia por conteo directo.

Determinación taxonómica de los HMA: Las preparaciones permanentes de las esporas de HMA se observaron en un microscopio óptico equipado con luz polarizada e iluminación Nomarski (Zeiss Mod 62223®) para examinar y contrastar las capas que componen 
la pared de las esporas y así determinar su consistencia, arreglo, reacción al reactivo de Melzer, presencia de ornamentaciones, escudos de germinación y tipo de hifa. Estas características morfológicas fueron la base para la determinación taxonómica de los HMA, considerando las descripciones especializadas de las especies reportadas a nivel mundial y disponibles en el sitio web http://www.amf-phylogeny. com/; así como las descritas en el manual de Schenck y Pérez (1990) y en la International Culture Collection of (Vesicular) Arbuscular Mycorrhizal Fungi (http://invam.wvu. edu/; INVAM, 2016). Para la nomenclatura taxonómica de los HMA se siguió la propuesta de Schüßler y Walker (2010), y se verificó en el Index Fungorum. Las preparaciones de referencia fueron depositadas en el Laboratorio de Biosistemática de Leguminosas, Departamento de Biología, Universidad Autónoma Metropolitana-Iztapalapa.

Análisis físico y químico del suelo: Los suelos de los siete sitios de las zonas cafetaleras en estudio fueron analizados, con la finalidad de tener información de las condiciones de fertilidad edáfica que permitieran interpretar y relacionar los resultados relativos a la abundancia, riqueza y composición de especies de HMA asociados con las plantas del café en cada sitio cafetalero. Los análisis se llevaron a cabo en el Laboratorio Institucional Acreditado, de Análisis de Suelos y Plantas de Ecosur, San Cristóbal, siguiendo la Nom-012-RECNAT-2000 (SEMARNAT, 2002). El pH activo del suelo se determinó con un potenciómetro equipado con un electrodo (Corning ${ }^{\circledR}$ ), en una suspensión de suelo: agua destilada en proporción 1:2 (peso/volumen). La conductividad eléctrica (CE) se determinó por extracto de saturación y filtración al vacío de una pasta de suelo saturada con agua destilada y se midió con un conductivímetro $\left(\right.$ Corning $\left.^{\circledR}\right)$. La materia orgánica del suelo (MO) se cuantificó por el procedimiento de Walkley y Black (1934). $\mathrm{El}$ fósforo disponible $\left(\mathrm{PO}_{4}^{-3}\right)$ se extrajo con $\mathrm{NaHCO}_{3}(\mathrm{pH}=8.5)$ y se determinó de acuerdo con Olsen, Cole, Watanabe y Dean (1954). La textura del suelo se determinó con el hidrómetro de Bouyoucos (Bouyoucos, 1962) y, por último, la capacidad de intercambio catiónico se calculó por el método del acetato de amonio (Rhoades, 1982). Todos los datos fueron expresados en peso seco de suelo.

Una prueba no paramétrica de KruskalWallis $(\mathrm{H})$ y una de medias por rangos se utilizaron para detectar diferencias en la abundancia de esporas, riqueza de morfoespecies y propiedades del suelo determinadas en los sitios de estudio. Un índice de disimilitud por distancias euclidianas con el método de Ward, basado en presencias-ausencias, fue usado para comparar la composición de especies de HMA entre los sitios; el índice es diferente de cero cuando hay completa disimilitud. La relación entre la estructura de las comunidades de HMA y las propiedades del suelo por sitio de cafetos fue explorada con un análisis de componentes principales (ACP). Los análisis se realizaron con el programa STATISTICA ver 6.0 y en todos los casos se usó una $\mathrm{P} \leq 0.05$.

\section{RESULTADOS}

Los sitios San Luis Nexapa, Platanar y Providencia registraron la mayor abundancia de esporas de HMA en $100 \mathrm{~g}$ de suelo seco $(\mathrm{H}=2.6, \mathrm{P} \leq 0.041)$, con valores promedio de 174258 y 273 , respectivamente; mientras que en los otros sitios presentaron menor abundancia con valores cercanos a las 100 esporas: 94 en San Agustín, 136 en 20 de noviembre, 96 en Victoria y 76 en Toluca.

El análisis taxonómico convencional de las esporas reveló la presencia de 20 morfoespecies de HMA pertenecientes a cuatro géneros: Acaulospora, Glomus, Gigaspora y Sclerocystis, además se detectó el género Scutellospora por la presencia de células auxiliares en el suelo. Los géneros más frecuentes fueron Acaulospora y Glomus, con diez y siete morfoespecies, respectivamente. Asimismo, algunos HMA como $S$. taiwanensi y A. laevis, registrados en cafetos de Toluca y Victoria fueron exclusivos de estos sitios (Cuadro 2). Para el estado de Chiapas se reportan por primera 
CUADRO 2

Hongos micorrizógenos arbusculares registrados en suelo rizosférico de Coffea canephora en siete sitios en fincas cafetaleras de la región del Soconusco, Chiapas, México

TABLE 2

Arbuscular mycorrhizal fungi recorded in rhizospheric soil of Coffea canephora at seven sites in coffee farms at the Soconusco region, Chiapas, Mexico

\begin{tabular}{|c|c|c|c|c|c|c|c|}
\hline \multirow[b]{2}{*}{ Especies de HMA } & \multicolumn{7}{|c|}{ Sitios de colecta } \\
\hline & $\begin{array}{l}\text { San } \\
\text { Agustín }\end{array}$ & $\begin{array}{l}20 \mathrm{de} \\
\text { noviembre }\end{array}$ & Toluca & $\begin{array}{l}\text { San Luis } \\
\text { Nexapa }\end{array}$ & Platanar & Providencia & Victoria \\
\hline Acaulospora sp. 1 & X & X & $\mathrm{X}$ & X & $\mathrm{X}$ & $\mathrm{X}$ & $\mathrm{X}$ \\
\hline Acaulospora sp. 2 & $\mathrm{X}$ & $\mathrm{X}$ & $\mathrm{X}$ & $\mathrm{X}$ & $\mathrm{X}$ & $\mathrm{X}$ & $\mathrm{X}$ \\
\hline Acaulospora excavata & & & $\mathrm{X}$ & & & $\mathrm{X}$ & $\mathrm{X}$ \\
\hline Acaulospora foveata* & & & $\mathrm{X}$ & & & & $\mathrm{X}$ \\
\hline Acaulospora laevis & & & & & & & $\mathrm{X}$ \\
\hline Acaulospora aff. mellea & & & $\mathrm{X}$ & $\mathrm{X}$ & & & $\mathrm{X}$ \\
\hline Acaulospora aff. morrowiae & $\mathrm{X}$ & & $\mathrm{X}$ & & $\mathrm{X}$ & $\mathrm{x}$ & $\mathrm{X}$ \\
\hline Acaulospora rehmii* & & & $\mathrm{X}$ & & & $\mathrm{X}$ & $\mathrm{X}$ \\
\hline Acaulospora scrobiculata & & & $\mathrm{X}$ & & $\mathrm{X}$ & & $\mathrm{X}$ \\
\hline Acaulospora spinosa & & & $\mathrm{X}$ & & & & $\mathrm{X}$ \\
\hline Glomus sp. 1 & $\mathrm{X}$ & $\mathrm{X}$ & $\mathrm{X}$ & $\mathrm{X}$ & $\mathrm{X}$ & $\mathrm{X}$ & $\mathrm{X}$ \\
\hline Glomus sp. 2 & & $\mathrm{X}$ & $\mathrm{X}$ & $\mathrm{X}$ & $\mathrm{X}$ & $\mathrm{X}$ & $\mathrm{X}$ \\
\hline Glomus sp. 3 & & & $\mathrm{X}$ & $\mathrm{X}$ & $\mathrm{X}$ & $\mathrm{X}$ & \\
\hline Glomus aff. ambisporum & & & & $\mathrm{X}$ & & & $\mathrm{X}$ \\
\hline Glomus fuegianum & & & & & & & $\mathrm{X}$ \\
\hline Glomus tortuosum* & & & $\mathrm{X}$ & & & & $\mathrm{x}$ \\
\hline Glomus trufemii* & & & $\mathrm{X}$ & & & & $\mathrm{x}$ \\
\hline Gigaspora aff. margarita & & & $\mathrm{X}$ & & $\mathrm{X}$ & & \\
\hline Sclerocystis sinuosa & & & $\mathrm{X}$ & & & & \\
\hline Sclerocystis taiwanensi* & & & $\mathrm{X}$ & & & & \\
\hline $\begin{array}{l}\text { Células auxiliares de } \\
\text { Scutellospora } \mathrm{sp} .\end{array}$ & & & & & & & $\mathrm{X}$ \\
\hline $\begin{array}{l}\text { Abundancia promedio } \dagger \\
\text { ( } \pm \text { error estand.) de esporas }\end{array}$ & $94( \pm 4)^{\mathrm{cb}}$ & $136( \pm 11)^{\mathrm{b}}$ & $76( \pm 6)^{c}$ & $174( \pm 16)^{\mathrm{a}}$ & $258( \pm 26)^{\mathrm{a}}$ & $273( \pm 14)^{\mathrm{a}}$ & $96( \pm 9)^{\mathrm{cb}}$ \\
\hline Riqueza de morfoespecies & 4 & 4 & 17 & 7 & 8 & 8 & 17 \\
\hline
\end{tabular}

*Nuevos registros para el estado de Chiapas, México / New records for Chiapas, Mexico.

$\dagger$ Letras distintas indican diferencias estadísticas significativas con $\mathrm{P} \leq 0.05$.

vez cinco nuevos registros de HMA, dos del género Acaulospora, dos de Glomus y uno de Sclerocystis (Cuadro 2, Fig. 2). Los sitios cafetaleros Toluca y Victoria registraron la mayor riqueza de HMA con 17 morfoespecies cada uno; en contraste los de San Agustín y 20 de noviembre tuvieron la menor riqueza con cuatro morfoespecies cada uno $(\mathrm{H}=2.1$, $\mathrm{P} \leq$ 0.036). Mientras que San Luis Nexapa, Platanar y Providencia presentaron valores intermedios de riqueza, con entre siete y ocho morfoespecies de HMA por sitio (Cuadro 2).

Los suelos de todos los sitios estudiados tienen un $\mathrm{pH}$ ácido que difirió entre sitios $(\mathrm{H}=$ $7.4, \mathrm{P} \leq 0.049$ ), con valores en el intervalo de 4.5 a 5.9 , con el valor más bajo registrado en el Platanar y el más alto en el suelo de 20 de noviembre. La CE del suelo fue baja sin diferencias entre los sitios. La capacidad de intercambio catiónico tampoco difirió entre los siete 

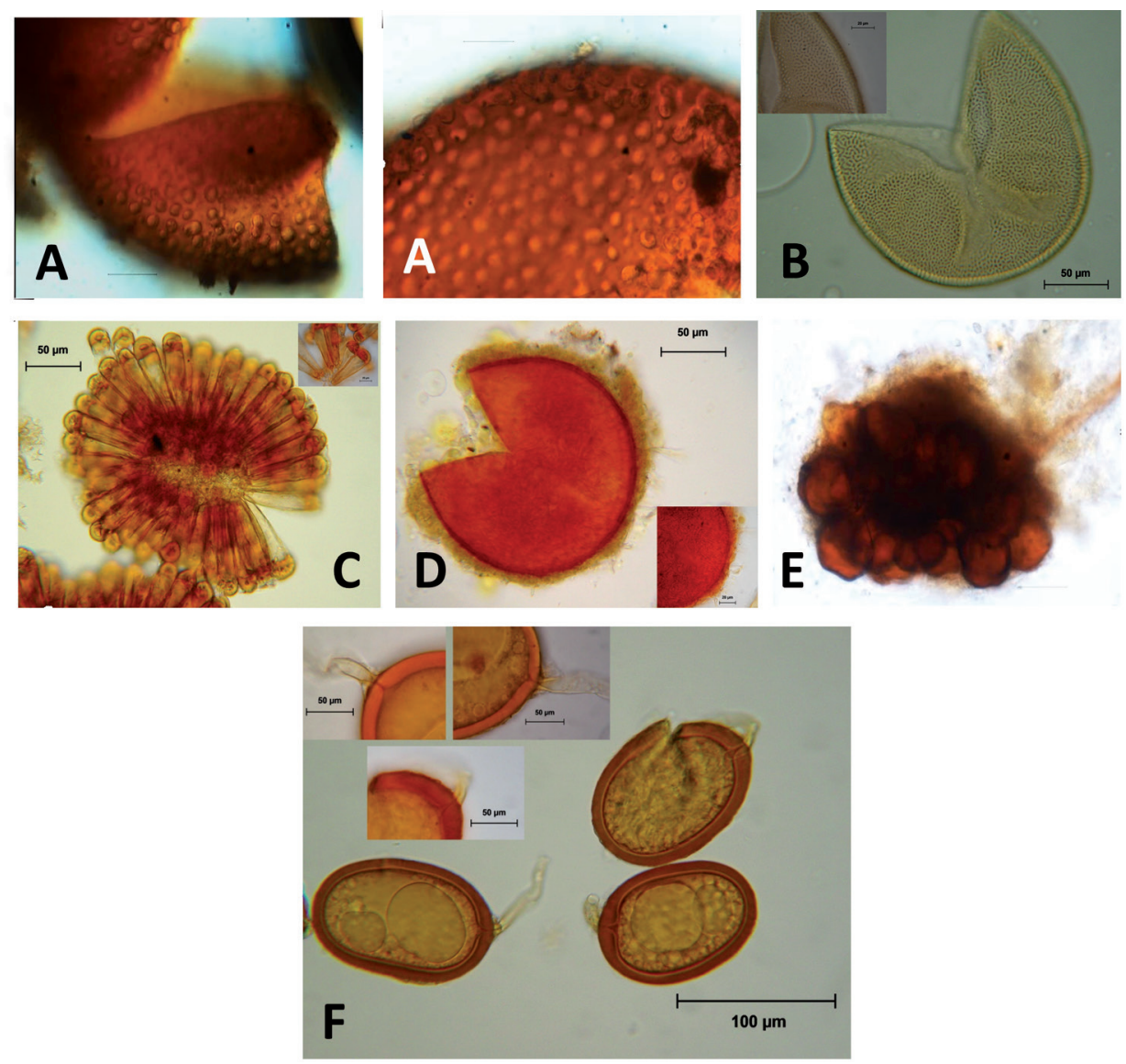

Fig. 2. Esporas de los nuevos registros de hongos micorrizógenos arbusculares. A. Acaulospora foveata, Janos \& Trappe; B. Acaulospora rehmii Sieverd. \& S. Toro; C. Sclerocystis taiwanensis C.G. Wu \& Z.C. Chen; D. Glomus tortuosum N.C. Schenck \& G.S. Sm.; E. Células auxiliares del género Scutellospora; F. Glomus trufemii B.T. Goto, G.A. Silva \& Oehl.

Fig. 2. Spores of new records of arbuscular mycorrhizal fungi. A. Acaulospora foveata, Janos \& Trappe; B. Acaulospora rehmii Sieverd. \& S. Toro; C. Sclerocystis taiwanensis C.G. Wu \& Z.C. Chen; D. Glomus tortuosum N.C. Schenck \& G.S. Sm.; E. Auxiliary cells of the genus Scutellospora; F. Glomus trufemii B.T. Goto, G.A. Silva \& Oehl.

sitios $(\mathrm{H}=9.6, \mathrm{P} \leq 0.12)$. Todos estos suelos son considerados de textura franca, la mayoría con un componente arenoso importante, con excepción de Victoria que tuvo mayor cantidad de arcilla. El P disponible varió entre los sitios $(\mathrm{H}=3.3, \mathrm{P} \leq 0.001)$. En Toluca y Victoria se detectó menor concentración de $\mathrm{PO}_{4}^{-3}$, en contraste con San Agustín, 20 de noviembre y San Luis Nexapa que presentaron mayor concentración de este nutriente en el suelo. En el caso de Providencia y Platanar, el $\mathrm{PO}_{4}^{-3}$ fue también menor, pero los valores registrados no fueron tan reducidos como en Toluca y Victoria. Los suelos de Toluca, 20 de noviembre, Platanar y Providencia registraron mayor concentración de MO que los de San Agustín, Victoria y San Luis Nexapa (Cuadro 3).

La composición HMA mostró que los suelos de Victoria y Toluca tuvieron una disimilitud del $97 \%$ con respecto a la composición de HMA en el suelo de los otros cinco sitios (Fig. 3). Por su parte, la composición de HMA en el suelo de los sitios Platanar y Providencia difirió en un $26 \%$ de aquella en San Luis Nexapa, 20 de noviembre y San Agustín, los cuales tuvieron entre ellos mayor similitud 
CUADRO 3

Características físicas y químicas del suelo recolectado en siete sitios en fincas cafetaleras de la región del Soconusco, Chiapas, México

TABLE 3

Physical and Chemical characteristics of soil collected at seven sites in coffee farms at the Soconusco region, Chiapas, Mexico

\begin{tabular}{|c|c|c|c|c|c|c|c|}
\hline Propiedades/Sitios & San Agustín & Victoria & Toluca & 20 de noviembre & Platanar & San Luis Nexapa & Providencia \\
\hline $\mathrm{pH}\left(\mathrm{H}_{2} \mathrm{O}\right)$ & $5.15^{\mathrm{a}}$ & $5.59^{\mathrm{a}}$ & $5.59^{\mathrm{a}}$ & $5.95^{\mathrm{a}}$ & $4.52^{\mathrm{b}}$ & $4.95^{\mathrm{ab}}$ & $4.83^{\mathrm{b}}$ \\
\hline $\mathrm{CE}\left(\mathrm{ds} . \mathrm{m}^{-1}\right)$ & 0.062 & 0.08 & 0.101 & 0.112 & 0.078 & 0.041 & 0.058 \\
\hline C.I.C (cmol. $\left.\mathrm{kg}^{-1}\right)$ & 33.97 & 38.37 & 36.9 & 33.97 & 36.9 & 33.97 & 38.37 \\
\hline $\mathrm{PO}_{4}^{-3}\left(\mathrm{mg} \cdot \mathrm{kg}^{-1}\right)$ & $29.32^{\mathrm{a}}$ & $0.8^{\mathrm{b}}$ & $0.8^{\mathrm{b}}$ & $23.23^{\mathrm{a}}$ & $2.23^{\mathrm{ab}}$ & $38.8^{\mathrm{a}}$ & $1.37^{\mathrm{ab}}$ \\
\hline MO (\%) & $6.16^{\mathrm{b}}$ & $9.56^{\mathrm{ab}}$ & $14.09^{\mathrm{a}}$ & $13.83^{\mathrm{a}}$ & $11.29^{\mathrm{a}}$ & $8.76^{\mathrm{ab}}$ & $13.22^{\mathrm{a}}$ \\
\hline Arena $(\%)$ & $59.6^{\mathrm{a}}$ & $29.6^{\mathrm{b}}$ & $47.6^{\mathrm{a}}$ & $33.6^{\mathrm{a}}$ & $63.6^{\mathrm{a}}$ & $52^{\mathrm{a}}$ & $50^{\mathrm{a}}$ \\
\hline Arcilla (\%) & $30.4^{\mathrm{a}}$ & $32.4^{\mathrm{a}}$ & $20.4^{\mathrm{b}}$ & $22.4^{\mathrm{b}}$ & $4.4^{\mathrm{c}}$ & $28^{\mathrm{ab}}$ & $30^{\mathrm{a}}$ \\
\hline Limo (\%) & $10^{\mathrm{bc}}$ & $38^{\mathrm{a}}$ & $32^{\mathrm{b}}$ & $44^{\mathrm{a}}$ & $32^{\mathrm{b}}$ & $20^{\mathrm{c}}$ & $20^{\mathrm{c}}$ \\
\hline Clase textural & $\begin{array}{l}\text { Franco } \\
\text { arenoso }\end{array}$ & $\begin{array}{c}\text { Franco } \\
\text { arcilloso }\end{array}$ & Franco & Franco & Franco arenoso & $\begin{array}{c}\text { Franco } \\
\text { arcilla arenoso }\end{array}$ & $\begin{array}{c}\text { Franco } \\
\text { arcilla arenoso }\end{array}$ \\
\hline
\end{tabular}

Nota: Letras distintas en la fila indican diferencias significativas a $\mathrm{P} \leq 0.05$; $\sin$ letra $=$ no significativo.

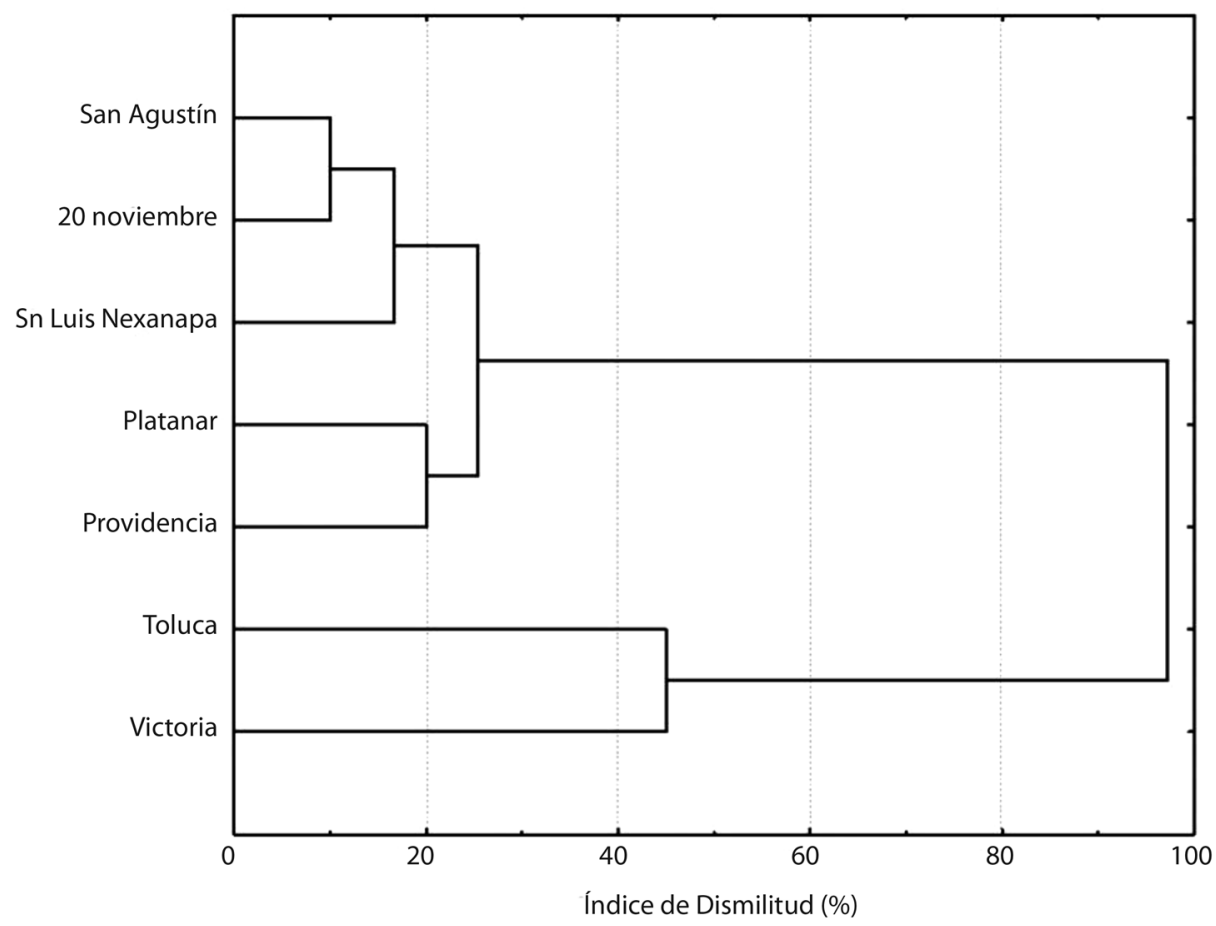

Fig. 3. Disimilitud en la composición de hongos micorrizógenos arbusculares en el suelo rizosférico de Coffea canephora entre siete sitios en fincas cafetaleras de la región del Soconusco, Chiapas, México.

Fig. 3. Dissimilarity in the composition of arbuscular mycorrhizal fungi in the rhizospheric soil of Coffea canephora among seven sites of coffee farms at the Soconusco region, Chiapas, Mexico. 
en la composición de HMA. El ACP, cuyos ejes principales explicaron el $62.5 \%$ de la varianza total (Fig. 4), ordenó a los sitios, a la abundancia de esporas y a la riqueza de HMA, con base en el P disponible y la CIC; en donde la riqueza de HMA correlacionó negativamente con el $\mathrm{PO}_{4}^{-3}(-0.699)$ y positivamente con la CIC (0.687). Asimismo, con respecto a la composición de HMA, el ACP indicó que algunos HMA como G. margarita o Glomus sp. 3 prefieren sitios con suelos arenosos (Toluca y Platanar) en comparación con Gl. ambisporum y Gl. fuegianum que al parecer prefieren suelos con mayor porcentaje de arcillas (e.g. Victoria).

\section{DISCUSIÓN}

Acaulospora foveata, Janos y Trappe está presente en varios ecosistemas de México (Montaño et al., 2012); sin embrago, no se tenía registro para el estado de Chiapas. A. foveata fue descrita por primera vez en 1982 en cultivos de caña de azúcar en el estado de Veracruz, así como en platanares y cacaotales de Costa Rica y Panamá (Janos \& Trappe, 1982; Trejo et al., 2015). Acaulospora rehmii Sieverd. y S. Toro se ha reportado en bosque tropical seco y húmedo sin perturbar; así como en agro-ecosistemas de México, entre ellos fincas cafetaleras en Veracruz (Trejo et al., 2011); no obstante, en Chiapas no se conocía, por lo que también es un nuevo registro para este estado. Sclerocystis taiwanensis C.G. Wu y Z.C. Chen es un HMA raro en México, se ha registrado en sistemas agroforestales de aguacate en Michoacán (Bárcenas, Varela, Stürmer, \& Chávez-Barcenas, 2011) y plantaciones de café en Veracruz México (Posada et al., 2016), por lo que su detección en la región del Soconusco indica que es un componente importante de la riqueza de HMA

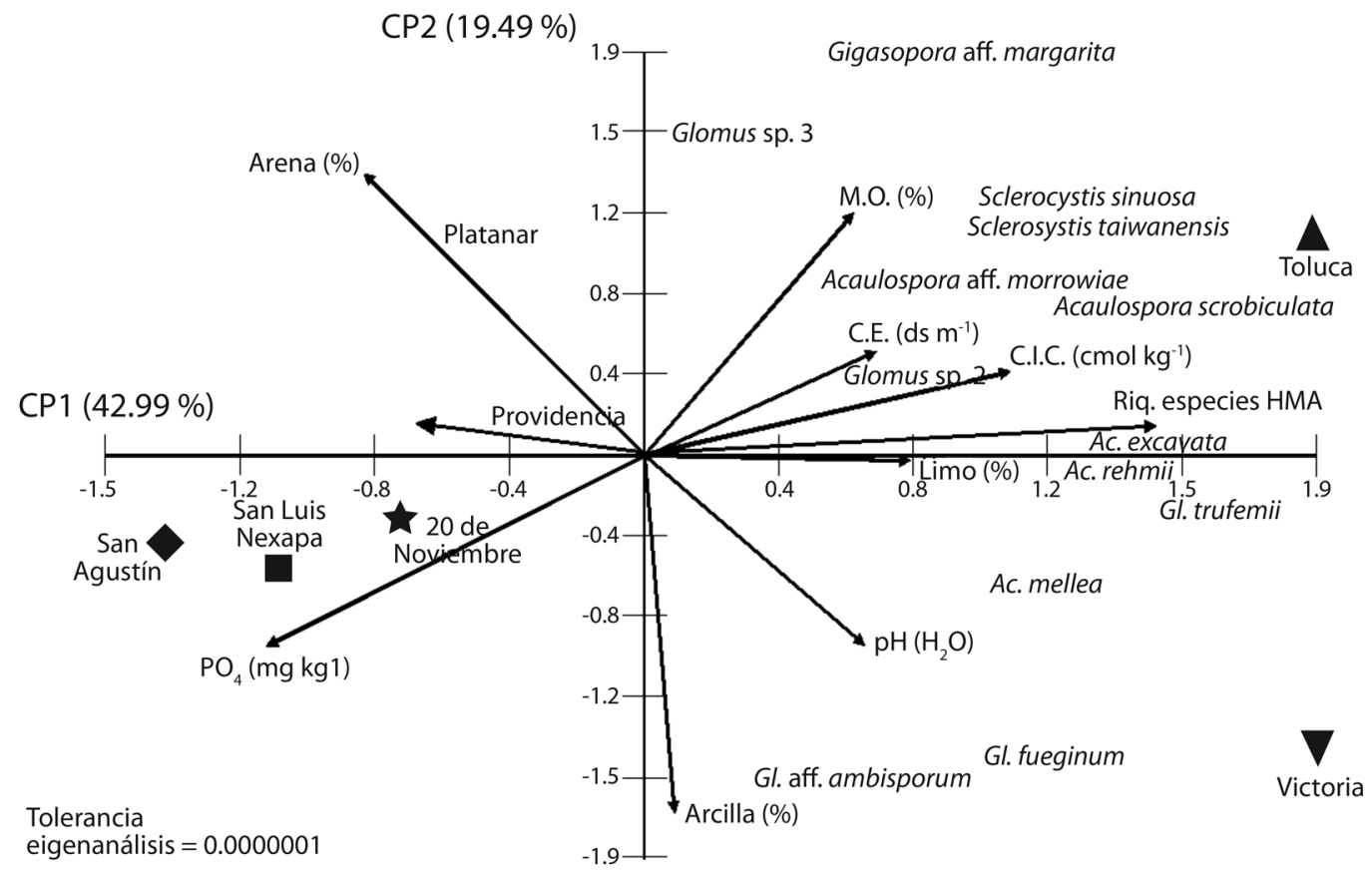

Fig. 4. Análisis de componentes principales que relaciona las propiedades del suelo (en vectores) con los hongos micorrizógenos arbusculares (Abundancia de esporas=AbEsp, composición de especies y riqueza de especies $=$ Riq. especies HMA) en el suelo rizosférico de Coffea canephora en siete sitios cafetaleros de la región del Soconusco, Chiapas, México. Fig. 4. Principal component analysis that correlates soil properties (in vectors) with arbuscular mycorrhizal fungi (abundance of spores=AbEsp, composition of species and species richness $=$ Riq. especies HMA) in the rhizospheric soil of Coffea canephora in seven sites with coffee plants at the Soconusco region, Chiapas, Mexico. 
en otros sistemas agroforestales cafetaleros en regiones tropicales húmedas. Así, este es el primer registro de $S$. taiwanensis para el estado de Chiapas; un HMA que forma esporas arregladas de forma radial en esporacarpos, similar a S. clavispora; sin embargo, difiere de esta especie en que los esporocarpos y las esporas son de menor tamaño y en el ápice de las esporas presentan una división central en forma de una depresión (Chi-Guang \& Zuei-Ching, 1987). Asimismo, Glomus tortuosum N.C. Schenck y G.S. Sm. se registra por primera vez para el estado de Chiapas, ya que en México se había documentado de otros ecosistemas tropicales secos y sistemas agroforestales con frutales (Montaño et al., 2012), y en particular no se había registrado en sistemas de cultivo de café (Arias et al., 2012; Trejo et al., 2011) y tampoco se conocía de otros ecosistemas de Chiapas (Violi et al., 2008; Pérez-Luna, Álvarez-Solís, Mendoza-Vega, Pat, Gómez-Álvarez, \& Hernández-Cuevas, 2012). En México, Glomus trufemii B. T. Goto, G. A. Silva y Oehl, se ha registrado recientemente en ecosistemas semiáridos en el estado de Oaxaca (Comunic. Pers., Hernández-Cuevas Laura). Esta especie se caracteriza por formar esporas subglobosas a elípticas $(<100$ micras $)$ dispuestas en esporocarpos laxos sin peridio, las esporas presentan una pared compuesta de dos capas, la más externa es hialina, semipermanente y está ausente en esporas maduras; la segunda capa es permanente, laminada, de color naranja rojizo y ninguna de las capas reacciona al reactivo de Melzer; además, la hifa se caracteriza por un cambio de coloración marcado en la base de las esporas. G. trufemii fue registrada por primera vez en dunas de Brasil (Tomio-Goto, Gomes, Alves Da Silva, Furrazola, Torres-Arias, \& Oehl, 2012). De esta manera, esta especie de HMA corresponde también al primer reporte para el estado de Chiapas y de manera oficial para México.

Existen patrones de interacción complejos entre las comunidades de HMA y de plantas, la riqueza y composición de HMA pueden diferir en función de los hospederos disponibles, la esporulación entre las especies de HMA y las condiciones edáficas (Smith \& Read, 2008). El manejo agronómico afecta negativamente a las comunidades de HMA con respecto a los sitios agrícolas que tienen una orientación orgánica (Oehl, Sieverding, Ineichien, Mader, Wiemken, \& Boller, 2009; de Beehouwer et al., 2015). En contraste, los sitios con manejo orgánico y vegetación nativa combinada con el cultivo del café incrementan la presencia de hospederos potenciales y generan condiciones edáficas particulares que benefician la relación de los hospederos con los HMA. Lo anterior, apoya la idea de que la riqueza de HMA se debe a la mayor diversidad de especies vegetales que caracterizan a los sistemas cafetaleros con baja y media tecnificación como ocurre en los sitios estudiados, misma que puede proveer más nichos ecológicos para las especies de HMA como se ha reportado en otras investigaciones (Chifflot, Rivest, Oliver, Cogliatro, \& Khasa, 2009; Trejo et al., 2011).

Es posible que la abundancia de esporas de HMA sea mayor en sistemas donde se utilizan especies arbóreas de manera intercalada en comparación con aquellos con monocultivo, o bien que se trate de sitios con vegetación en un estado de perturbación o madurez intermedios y con hospederos dominantes que pudieran estar promoviendo la producción de esporas como se ha reportado en plantaciones de banano (Mahecha-Vásquez, Sierra, \& Posada 2017). Esto ayudaría a explicar los valores intermedios de riqueza de morfoespecies de HMA en los cafetos de sitios como San Luis Nexapa, Platanar y Providencia, los cuales a su vez presentan mayor abundancia de esporas y una composición de morfoespecies intermedia. Esta idea podría estar apoyada en la hipótesis de que a niveles intermedios de perturbación la riqueza específica se ve favorecida, posiblemente porque en los ecosistemas menos perturbados, dominan especies mejor adaptadas y más competitivas (Roxburgh, Shea, \& Wilson, 2004), las cuales podrían favorecer a los HMA como se ha reportado en otras plantaciones tropicales (Mahecha-Vásquez et al., 2017). No obstante, es necesario explorar más sitios estratégicos y desarrollar metodologías más 
sensibles para la detección de todas las especies de HMA, ya que no se descarta la posibilidad de que las morfoespecies identificadas de HMA puedan estar subestimadas, debido a la dificultad de caracterizar aquellos HMA no esporulantes o en estado de latencia.

La alta riqueza y la distinta composición de especies de HMA asociadas a los cafetos de Victoria y Toluca en relación con otros sitios como San Agustín, 20 de noviembre y San Luis Nexapa, podrían atribuirse a que los suelos de Victoria y Toluca registraron menos $\mathrm{P}$ disponible. Varios estudios muestran que las plantas en general (Smith, Jakobsen, Gronlun, \& Smith, 2011), incluyendo las de café (Vaast et al., 1997; Andrade et al., 2009; Trejo et al., 2011), requieren de establecer una simbiosis funcional con los HMA cuando la disponibilidad del $\mathrm{P}$ es reducida, ya que la micorriza arbuscular facilita el acceso de las raíces de la planta hospedera a nutrientes con poca movilidad en el suelo (Smith \& Read, 2008; Cuenca, 2015), aspecto que es frecuente en suelos moderadamente ácidos (4.5 - 6). Un pH ácido promueve la fijación química del $\mathrm{PO}_{4}^{-3}$ con el aluminio $\left(\mathrm{Al}^{+3}\right)$, lo que reduce la disponibilidad de este nutriente en el suelo (Bünemann, Oberson, \& Frossard, 2011) y se ha visto que en estas condiciones es frecuente la presencia de HMA de los géneros de Acaulospora y Ambispora (Castillo, Borie, Godoy, Rubio, \& Sieverding, 2006; Cuenca, 2015; Trejo et al., 2015; Posada et al., 2016), lo que explicaría la dominancia de Acaulospora en los cafetos examinados en este estudio y resalta la importancia de éste género en las comunidades de HMA en condiciones de baja disponibilidad de $\mathrm{P}$ en el suelo. Asimismo, debido a que estos géneros de HMA se reportan también en suelos con alto $\mathrm{P}$ disponible, pero con $\mathrm{pH}$ ácido, es posible que en los sitios de cafeto examinados en este trabajo, el $\mathrm{pH}$ promueva de forma importante la presencia de estos géneros y de la riqueza de HMA como ocurre en otras plantaciones (Mahecha-Vásquez et al., 2017).

En el sentido anterior, es necesario destacar que la alta abundancia de esporas y los valores intermedios de riqueza de especies de
HMA en los cafetos de Providencia y Platanar podrían también estar asociados a una baja disponibilidad de $\mathrm{P}$ en el suelo; sin embargo, las concentraciones de $\mathrm{PO}_{4}^{-3}$ detectadas podrían sugerir un intervalo de disponibilidad menos drástico en estos sitios, lo que permite la presencia de algunos HMA también registrados tanto de los sitios con mayor como con menor P disponible en el suelo. Vaast et al. (1997) indican que algunas especies de HMA tienen una mejor respuesta a determinados niveles de $\mathrm{P}$ disponible que otras, como resultado de su diferente capacidad para capturar este nutriente del suelo. Por su parte, la relación positiva detectada entre los HMA y la CIC sugiere, por un lado, que la adquisición de formas catiónicas de otros nutrientes podría no depender de la asociación entre los HMA y los cafetos en la región del Soconusco, en donde las arcillas y la MO estarían probablemente aportando estos nutrientes; y por el otro, que un fuerte remplazo del $\mathrm{Al}^{+3}$ podría estar ocurriendo en la superficies de intercambio catiónico del suelo, promoviendo su solubilización y combinación con el $\mathrm{PO}_{4}^{-3}$ para formar compuestos insolubles que reducen la disponibilidad del P (Bünemann et al., 2011). No obstante, si bien la asociación negativa entre el $\mathrm{P}$ del suelo y el desarrollo de la micorriza arbuscular es un aspecto que ha sido ampliamente documentado, no hay reportes del efecto de los nutrientes catiónicos del suelo sobre los HMA.

En conjunto, los resultados de este estudio muestran que la disponibilidad del P, ligada a la acidez del suelo, podría estar regulando las comunidades de HMA en el suelo de la rizosfera de los cafetos en la región del Soconusco, Chiapas, lo que coincide con otras investigaciones que indican la relevancia de las características del suelo sobre los HMA en otras plantaciones de café (de Beehouwer et al., 2015, Posada et al., 2016). Así, se sugiere que podrían haber consorcios de HMA específicos para los niveles de $\mathrm{P}$ y acidez presentes en diferentes sitios con cafetos, o bien consorcios de especies de Acaulospora y Glomus que pudieran ser comunes para las condiciones ambientales en las que se desarrolla el café, 
cuya identificación y compatibilidad funcional debe ser examinada antes de emplearse como biofertilizantes dirigidos a favorecer el desarrollo y rendimiento de los cafetos, como lo reportan otros estudios (Aguirre-Medina, 2006; Trejo et al., 2011; Adriano et al., 2011).

En conclusión, los diferentes sitios con cultivo de café examinados en este estudio contienen cinco nuevos registros para el estado de Chiapas y una importante riqueza de HMA, cuya dinámica podría depender de la disponibilidad de $\mathrm{P}$ y acidez en el suelo. Los HMA del género Acaulospora parecen ser los más frecuentemente registrados bajo las condiciones edáficas de la época de secas en las que crece Coffea canephora en la región del Soconusco, Chiapas; no obstante, es necesario examinar el efecto que pudieran tener las diferentes especies HMA registradas para la familia Glomeraceae; ya que en conjunto pudieran tener influencia positiva sobre el desarrollo y rendimiento de esta variedad de café; esta información permitiría seleccionar aislados y/o consorcios de HMA para la obtención de biofertilizantes nativos aplicables al cultivo de Coffea canephora en esta región de México.

\section{AGRADECIMIENTOS}

Al proyecto "Diseño, equipamiento y puesta en marcha de un Centro Estatal de Innovación y Transferencia Tecnológica para el Desarrollo de la Cafeticultura Chiapaneca" (FOMIX\#249930) del Instituto del Café del estado de Chiapas, México y al proyecto "Multidisciplinario y Transversal Innovación Socio-ambiental en Zonas Cafetaleras para la Reducción de la Vulnerabilidad" (MT\#1106311262) de El Colegio de la Frontera Sur, México. L. Varela agradece a la UAMIztapalapa por otorgarle la Cátedra Divisional Ramón Riba y Nava Esparza.

\section{RESUMEN}

El cultivo del café, en la economía mexicana y en la región del Soconusco en Chiapas, aporta importantes divisas; $\sin$ embargo es susceptible a plagas y enfermedades, por lo que se requiere reforzar su producción a través de un manejo más sustentable. Este estudio analizó la abundancia, riqueza y composición de hongos micorrizógenos arbusculares (HMA) nativos que pudieran emplearse en un futuro como biofertilizantes en el cultivo del café. En 21 muestras de suelo recolectadas en noviembre 2015 de siete sitios en la región del Soconusco, Chiapas, México, cultivados con café "robusta" (Coffea canephora), se cuantificó la abundancia de esporas, la riqueza y composición de morfoespecies de HMA y se exploró su relación con las propiedades del suelo. Se obtuvo un total de 20 morfoespecies y cinco nuevos registros de HMA para Chiapas, las morfoespecies más frecuentes pertenecen a los géneros Acaulospora y Glomus. Los sitios Toluca y Victoria tuvieron mayor riqueza de especies (17 spp. c/u) que San Agustín, 20 de noviembre y San Luis Nexapa (4 - 7 spp. $\mathrm{c} / \mathrm{u}$ ); mientras que Providencia y Platanar registraron una riqueza intermedia y los mayores valores de abundancia de esporas. Los sitios Victoria y Toluca presentaron un $97 \%$ de disimilitud en la composición de especies de HMA con respecto a los demás sitios; la cual fue explicada por la baja concentración de $\mathrm{PO}_{4}^{-3}$ en el suelo de Victoria y Toluca. La disponibilidad de $\mathrm{P}$, ligada a la acidez del suelo, fueron los factores que podrían estar regulando las comunidades de HMA en el suelo de la rizosfera de los cafetos en la región del Soconusco. Se propone que podrían haber consorcios de HMA específicos para los niveles de $\mathrm{P}$ y acidez presentes en diferentes sitios con cafetos, o bien consorcios de especies de Acaulospora y Glomus acordes con las condiciones ambientales en las que se desarrolla el café, cuya compatibilidad y funcionalidad debe examinarse antes de emplearlos como biofertilizantes nativos en los cafetos de esta región tropical de México.

Palabras clave: esporas fúngicas, fósforo del suelo, micorriza arbuscular, plantaciones de café.

\section{REFERENCIAS}

Adriano, Ma. L., Jarquín, R., Hernández, C., Figueroa, M. S., \& Monreal, C. T. (2011). Biofertilización de café orgánico en etapa de vivero en Chiapas, México. Revista Mexicana de Ciencias Agrícolas, 2(3), 417-431.

Aguirre-Medina, J. F. (2006). Biofertilizantes microbianos: Experiencias agronómicas del programa nacional del INIFAP en México (Núm. 2, pp. 201). Campo Experimental Rosario Izapa. Chiapas, México: Instituto Nacional de Investigaciones Forestales, Agrícolas y Pecuarias. Centro de Investigaciones Regionales Pacífico Sur.

Andrade, S., Mazzafera, P., Schiavinato, M., \& Silveira, A. (2009). Arbuscular mycorrhizal association in coffee. Journal of Agricultural Science, 147, 105-115.

Arias, R. M., Heredia, G., Sosa, V. J., \& Fuentes-Ramírez, L. E. (2012). Diversity and abundance of arbuscular 
mycorrhizal fungi spores under different coffee production systems and in a tropical montana cloud forest patch in Veracruz, Mexico. Agroforestry Systems, 85,179-193.

Barrera, J. F., \& Parra, M. (2000). El café en Chiapas y la investigación en ECOSUR. Ecofronteras, 12, 3-6.

Bárcenas, A., Varela, L., Stürmer, S. L., \& ChávezBárcenas, A. T. (2011). Catálogo de hongos micorrizógenos arbusculares de huertos de aguacate de Michoacán, México. Proceedings VII World Avocado Congress, Australia.

Bouyoucos, G. J. (1962). Hydrometer improved method for making particles size analysis of soil. Agronomy Journal, 54, 464-465.

Bünemann, E. K., Oberson, A., \& Frossard, E. (2011). Phosphorus in Action: Biological Processes in Soil Phosphorus Cycling (Soil Biology). Berlin, Alemania: Springer-Verlag.

Brundrett, M., Bougher, N., Dell, B., Grove T., \& Malajczuk, N. (1996). Working with mycorrhizal in forestry and agriculture. Canberra, Australia: Australian Centre for International Agricultural Research, Monograph.

Castillo, C. G., Borie, F. R., Godoy, R., Rubio, R., \& Sieverding, E. (2006). Diversity of arbuscular mycorrhizal plant species and fungal species in evergreen forest, deciduous forest and grassland ecosystems of Southern Chile. Journal of Applied Botany Food Quality, 80, 40-47.

CEDRSSA (Centro de Estudios para el Desarrollo Rural Sustentable y la Soberanía Alimentaria). (2014). Producción y mercado de café en el mundo y en México. Recuperado de http://www.cedrssa.gob.mx/includes/ asp/download.asp?iddocumento $=2756 \&$ idurl $=4576$

Chi-Guang, W., \& Zuei-Ching, C. (1987). The Endogonaceae of Taiwan, II. Two new species of Sclerocystis from Taiwan. Transactions of the Mycological Society, 2(2), 73-83.

Chifflot, V., Rivest, D., Olivier, A., Cogliatro, A., \& Khasa, D. (2009). Molecular analysis of arbuscular mycorrhizal community structure and spores distribution in tree-based intercropping and forest systems. Agriculture, Ecosystems and Environment, 131, 32-39.

CONANP (Comisión Nacional de Áreas Naturales Protegidas). (2011). Estudio Previo Justificativo para la Modificación de la Declaratoria de la Reserva de la Biosfera Volcán Tacaná, Chiapas, México. Comisión Nacional de Áreas Naturales Protegidas. Recuperado de www.conanp.gob.mx

Cuenca, G. (2015). Las micorrizas arbusculares: aspectos teóricos y aplicados. Caracas, Venezuela: Ediciones IVIC, Instituto venezolano de Investigaciones Científicas.
Cuenca, G., \& Lovera M. (2010). Seasonal variation and ditribution at different soil depth of arbuscular mycorrhizal fungi spore in a tropical sclerophyllous shrubland. Botany, 88(1), 54-64.

de Almeida, V. C., Nogueira, M. I., Guimaraes, R. J., \& Mourao Jr., M. (2003). Carbono da biomassa microbiana e micorriza en solo sub mata nativa e agroecosistemas cafeeiros. Acta Sciences Agronomica, 25, 147-153.

de Beenhouwer, M., Van Geel, M., \& Ceulemans T., Muleta D., Lievens, B., Honnay, O. (2015). Changing soil characteristics alter the arbuscular mycorrhizal fungi communities of Arabica coffee (Coffea arabica) in Ethiopia across a management intensity gradient. Soil Biology \& Biochemistry, 91,133-139.

DGSV-CNRF (Dirección General de Sanidad VegetalSAGARPA, SENASICA). (2015). Programa de Vigilancia Epidemiológica Fitosanitaria de la Roya del Café 2013-2015. Recuperado de http://royacafe. lanref.org.mx/index.php.

FAO (Food and Agriculture Organization of the United Nations). (2015). Manejo Agroecológico de la Roya del Café (pp. 80). Memorias del Seminario Científico Internacional. Ciudad de Panamá, Panamá: FAO.

Flores, F. V. (2015). La producción de café en México: ventana de oportunidad para el sector agrícola de Chiapas. Espacio $I+D$, innovación más desarrollo, 4(7), 175-194.

Fonseca, A. (2006). El café de sombra: un ejemplo de pago de servicios ambientales para proteger la biodiversidad. Gaceta Ecológica, 80, 19-31.

Gerdemann, J. W., \& Nicolson, T. H. (1963). Spores of mycorrhizal Endogone species extracted from soil by wet sieving and decanting. Transactions of the British Mycological Society, 46, 235-244.

Heredia, G., \& Arias, R. M. (2008). Hongos saprobios y endomicorrizógenos en suelos. En R. H. Manson, V. Hernández-Ortiz, S. Gallina, \& K. Mehltreter (Eds.), Agroecosistemas cafetaleros de Veracruz: biodiversidad, manejo y conservación (pp. 193-212). Jalapa, México: Instituto de Ecología A.C. (INECOL) e Instituto Nacional de Ecología (INE-SEMARNAT).

Hijmans, R. J., Cameron, S. E., Parra, J. L., Jones, P. G. \& Jarvis, A. (2005). Very high resolution interpolated climate surfaces for global land areas. International Journal of Climatology, 25, 1965-1978.

ICO (International Coffee Organization). (2016a). Trade Statistics Tables. Exports of coffee by exporting countries. Recuperado de http://www.ico.org/trade_statistics.asp

ICO (International Coffee Organization). (2016b). Historical Data on the Global Coffee Trade. Total production-Crop Year. Recuperado de http://www.ico. 
org/historical/1990\%20onwards/PDF/1a-total-production.pdf

INIFAP (Instituto Nacional de Investigaciones Forestales y Agropecuarias). (2010). Uso de suelo y vegetación 1:1000000. Comisión Nacional para el Conocimiento y Uso de la Biodiversidad, México D.F. Recuperado de www.conabio.gob.mx

INVAM (International Culture Collection of (Vesicular) Arbuscular Mycorrhizal Fungi). (2016). West Virginia University. Recuperado de http://invam.wvu.edu/

Janos, D. P., \& Trappe, J. M. (1982). Two new Acaulosporas from Tropical America. Mycotaxon, 15, 515- 522.

Mahecha-Vásquez, G., Sierra, S., \& Posada, R. (2017). Diversity indices using arbuscular mycorrhizal fungi to evaluate the soil state in banana crops in Colombia. Applied Soil Ecology 109, 32-39.

Mariscal, A. (2011). El café orgánico de Chiapas crece a contracorriente y sin incentivo. CNN México. Recuperado de http://expansion.mx/nacional/2011/01/25/ el-cafe-organico-de-chiapas-crece-a-contracorriente$y$-sin-incentivos

Moguel, P. \& Toledo, V. (1999). Biodiversity conservation in traditional coffee systems of Mexico. Conservation Biology, 13(1), 11-21.

Montaño, N. M., Alarcón, A., Camargo-Ricalde, S. L., Hernández-Cuevas, L. V., Álvarez-Sánchez, J., González-Chávez, ... Ferrera-Cerrato, R. (2012). Research on arbuscular mycorrhizae in Mexico: an historical synthesis and future prospects. Symbiosis, 57, 111-126.

Oehl, F., Sieverding, E., Ineichen, K., Mader, P., Wiemken, A., \& Boller, T. (2009). Distinct sporulation dynamics of arbuscular mycorrhizal fungal communities from different agroecosystems in long-term microcosms. Agriculture, Ecosystems \& Environment, 134, 257-268.

Olsen, S. R., Cole, C. V., Watanabe, F. S., \& Dean, L. A. (1954). Estimation of available phosphorus in soils by extraction with sodium bicarbonate. USDA circ. 939. Washington D.C., EEUU: US Government Printing Office.

Pérez-Luna, Y. C., Álvarez-Solís, J. D., Mendoza-Vega, J., Pat, J. M., Gómez-Álvarez R., \& Hernández-Cuevas, L. V. (2012). Diversidad de hongos micorrícicos arbusculares en maíz con cultivo de cobertura y biofertilizantes en Chiapas, México. Gayana Botánica, 69(1), 46-56

Posada, R. H., Sánchez de Prager, M., Heredia, G., \& Sieverding, E. (2016). Effects of soil physical and chemical parameters, and farm management practices on arbuscular mycorrhizal fungi communities and diversities in coffe plantations in Colombia and Mexico. Agroforestry Systems, DOI 10.1007/ s10457-016-0030-0

Rhoades, J. (1982). Cation Exchange Capacity. En A. L. Page (Ed.). Methods of Soil Analysis. Part 2. Agronomy Monograph No 9 (pp. 178-190) Madison, EEUU: ASA \& SSSA Press.

Roxburgh, S. H., Shea, K., \& Wilson, J. B. (2004). The intermediate disturbance hypothesis: Patch dynamics and mechanisms of species coexistence. Ecology, 85(2), 359-371.

Secretaria de Medio Ambiente y Recursos Naturales (SEMARNAT). (2002, 31 de diciembre). Norma oficial mexicana NOM-021-SEMARNAT-2000. Diario Oficial, pp. 1-85.

SHCP (Secretaría de Hacienda y Crédito Público). (2014). Panorama del café. Recuperado de http://www. financierarural.gob.mx/informacionsectorrural/Panoramas/Ficha $\% 20 \mathrm{Caf} \% \mathrm{C} 3 \% \mathrm{~A} 9$.pdf

Smith, S. E., Jakobsen, I., Gronlund, M., \& Smith, A. (2011). Roles of arbuscular mycorrhizas in plant phosphorus nutrition: Interactions between pathways of phosphorus uptake in arbuscular mycorrhizal roots have important implications for understanding and manipulating plant phosphorus acquisition. Plant Physiology, 156, 1050-1057.

Smith, S. E., \& Read, D. J. (2008). Mycorrhizal symbiosis. San Diego, EEUU: Academic Press.

Schenck, C. N., \& Pérez, Y. (1990). Manual for the identification of VA mycorrhizal fungi. Gainesville, EEUU: Synergistic Publications.

Schüßler, A., \& Walker, C. (2010). Glomeromycota species list. Recuperado de http://www.lrz.de/ schuessler/ amphylo/

Tomio-Goto, B., Gomes, J. J., Alves Da Silva, G., Furrazola, E., Torres-Arias, Y., \& Oehl, F. (2012). Glomus trufemii (Glomeromycetes), a new sporocarpic species from Brazilian sand dunes. Mycotaxon, 120, 1-9.

Trejo, D., Ferrera-Cerrato, R., García, R., Varela, L., Lara, L., \& Alarcón A. (2011). Efectividad de siete consorcios nativos de hogos micorrízicos arbusculares en plantas de café en condiciones de invernadero y campo. Revista Chilena de Historia Natural, 84, 23-31.

Trejo, D., Guzmán, G., Lara, L., Zulueta, R., Palenzuela, J., Sánchez-Castro, I., ... Oeh, F. (2015). Morphology and phylogeny of Acaulospora foveata (Glomeromycetes) from Mexico. Sydowia, 67, 119-126. 
Vaast, P., Zasoski, R. J., \& Bledsoe, C. S. (1997). Effects of vesicular-arbuscular mycorrhizal inoculation at different soil $\mathrm{P}$ availabilities on growth and nutrient uptake of in vitro propagated coffee (Coffea arabica L.) plants. Mycorrhiza, 6, 493-497.

Van der Heijden, M. G. A., \& Sanders, I. R. (2002). Mycorrhizal Ecology. Berlin, Alemania: Springer-Verlag.

Violi, A. H., Barrientos-Priego, A. F., Wright, S. F., Escamilla-Prado, E., Morton, J. B., Menge, J. A., \&
Lovatt, C. J. (2008). Disturbance changes arbuscular mycorrhizal fungal phenology and soil glomalin concentrations but not fungal spore composition in montane rainforests in Veracruz and Chiapas, México. Forest Ecology \& Management, 254, 276-290.

Walkley, A., \& Black, I. A. (1934). An examination of the Degtjareff method for determining soil organic matter and a proposed modification of the chromic acid titration method. Soil Science, 37, 29-38. 